\title{
Commentary
}

\section{Understanding the social determinants of behaviours: Can new methods help?}

\author{
Ana V. Diez Roux ${ }^{\mathrm{a}, *}$, Amy H. Auchincloss ${ }^{\mathrm{b}}$ \\ a Center for Integrative Approaches to Health Disparities, University of Michigan, United States \\ ${ }^{\mathrm{b}}$ Department of Epidemiology, Drexel University, United States
}

An individual's behaviour undoubtedly results from a set of complicated processes involving interactions between environmental factors, personal characteristics, and biology. Moreover it is clear that social processes, both through their influence on the social and physical environments in which people live and work, as well as through the transmission of norms and attitudes through social networks, play a key role in shaping behaviours. Understanding these social processes and the ways in which they affect behaviour is fundamental to the identification of the most effective interventions to improve health and reduce inequalities in health. The papers by Galea, Hall, \& Kaplan (this issue) and Cooper, Bossak, Tempalski, Des Jarlais, and Friedman (this issue) emphasize the need to study these social determinants in the specific case of drug use. More specifically, they discuss important methodological challenges involved and suggest and illustrate the application of new methods. Cooper et al. focus on the measurement of spatially defined environments and Galea et al. focus on use of agent-based models to better capture how relations between individuals affect population-level patterns of drug use.

A first step in studying the social determinants of health behaviours is the development of theories regarding the relevant social factors and the ways in which they may operate. Although a specific methodology is not a prerequisite for the development of theory, the use of a specific analytical approach can influence the types of theories that are developed because the analytical methods determine what can be tested and what cannot. For this reason, the advent of new methods may sometimes stimulate new theories. For example, the advent of multilevel analysis stimulated much needed theorizing on the ways in which group-level or contextual factors may affect health. The geographical and spatial methods illustrated by Cooper et al. may stimulate more sophisticated theorizing on the ways in which space shapes the distribution of health related outcomes. The dynamic agent models illustrated by Galea et al. may stimulate theorizing on the ways in which dynamic interactions between people, and between people and their environments affect behaviours and health outcomes. One of the challenges to epidemiologic research today is that the excessive reliance on a

\footnotetext{
* Corresponding author at: Department of Epidemiology, 109 Observatory Street, SPH Tower, Rm 3671, Ann Arbor, MI 48109, United States. Tel.: +1 734615 9204; fax: +1 7349980006 .

E-mail address: adiezrou@umich.edu (A.V. Diez Roux).
}

single analytical approach (exemplified by the statistical analysis of data) has to a certain extent impoverished our thinking and theory development, a subject we will return to later.

Another key challenge in studying the social determinants of health is measurement. Health researchers are woefully unsophisticated at measuring the attributes of social groups or contexts. Only recently has interest in this area grown (spurred in part by the advent of multilevel analysis) and generated interesting methodological approaches (Ali et al., 2002; Mujahid, Diez Roux, Morenoff, \& Raghunathan, 2007; Raudenbush \& Sampson, 1999). Geographic or spatial contexts such as those investigated by Cooper et al. are only one of the many social contexts that may be relevant to health. As Galea et al. illustrate, features of social networks and the information and norms that flow through them are another example. Other examples include broader society or population level factors including the presence of inequality, macroeconomic conditions, and policy features. Measurement is fundamental not only because what is not measured cannot be studied empirically but also because good measurement requires a clear a priori specification of the construct. For this reason, good (and scientifically relevant) measurement is strongly linked to good theories. In addition, the measurement of environments broadly understood to encompass both physical and social attributes is fundamental to the identification of the often hypothesised (although less often documented) gene-environment interactions which are believed to be involved in virtually all diseases. Unfortunately the "environment" in gene-environment interaction research is sometimes reduced exclusively to the very proximate biologic environment as in the case of biomarkers of environmental exposures. But levels of these biomarkers are themselves the product of gene-environment interactions. The study of gene-environment interactions requires taking the environment much more seriously. For these reasons the development of sophisticated measurement strategies for geographically and non-geographically bounded contexts is key.

In their paper Cooper et al. provide examples of the measurement of attributes of a geographically bounded context relevant to drug use. They draw on the tools of geography to construct measures of spatial access to syringe exchange programmes and spatial exposure to criminal justice activity. They illustrate some of the complexities involved in linking spatially referenced data. Geographic Information Systems have now made it possible to create flexible spatial access measures that allow researchers to investigate spatial contexts of different definition and size. 
However, identifying the spatial context relevant for a specific process remains an important difficulty in the study of the effects of geographic contexts on health.

A third challenge has to do with analytical approaches. Essentially, the ultimate objective of most of the analytical techniques used in health research is the manipulation of observational data in order to approximate what would have been observed in an experiment in which persons are randomly assigned to the factor of interest in order to determine whether this factor has a causal effect on the outcome. This manipulation essentially involves the use of statistical techniques such as regression to approximate the counterfactual comparison of interest, that is, the comparison of the outcome in unexposed subjects to what would have been observed in the same subjects had they been exposed. These statistical approaches attempt to isolate the association of interest holding all other factors constant. Among other things, this approach assumes that effects are additive (on the log or linear scales), that there are no feedback loops or non-recursive relationships, and that outcomes for two individuals are independent conditional on other variables in the model (including for example random effects in multilevel models). Of course no one believes that this is the way the real world works. But the purpose of any model is to simplify reality so that it is tractable. The question is whether this simplification ignores fundamental aspects and causes us to arrive at the wrong answer to our question.

Epidemiologists and other health researchers have long recognized that health both at the individual and at the populationlevel is the outcome of the functioning of a system which involves dynamic relations between factors, between individuals, and between individuals and environments (Diez Roux, 1998; Koopman, 1996; Loomis \& Wing, 1990; Stallones, 1973). Arguably, current analytical approaches may lead us to incorrectly estimate the "causal effect" of a factor or the impact of changing a factor if they do not consider these dynamic relations. For example it could be argued that the impact of reducing the availability of a drug on drug use cannot be properly estimated without considering the dynamic relations between drug users and between the presence of drug users and the location of sellers. Ignoring these relations could lead us to the wrong answer. Dynamic models (of which the agentbased models illustrated by Galea et al. are one example) would allow us to estimate these associations while accounting for these complex relations, thus arriving at a more accurate assessment of the results we might expect by intervening on these factors in the real world (Auchincloss \& Diez Roux, 2008; Koopman, 2004; Page, 2008).

Galea et al. sketch a simple agent-based model to examine how drug dependence rates for different subgroups of the population vary as a function of changes in the degree of network (friend) influences on behaviour. They also examine whether the effects of network influences on dependence vary depending on the addictiveness of the drug, and whether the degree of network heterogeneity modifies the network effects. For example, using their model Galea et al. illustrate how at low levels of network influence, the impact of increasing network influence on dependence rates differs depending on spatial access to sellers. At higher levels of network influence, greater network influence is associated with less dependence in all groups because the predominant behaviour in the network is always non-dependence (hence by virtue of the rules written into the model everyone's behaviour shifts towards the most common behaviour).

A well known issue in simulation modelling is that all results are contingent on model input: in this specific example, the specific spatial placements of sellers and persons of different types, as well as on the specific rules by which access to drugs, prior use of drugs, and use of drugs among friends affects the drug dependence for a given individual by a certain magnitude. Because their report is intended as a simple illustration, Galea et al. do not provide details on the specific parameters in their model or on how results changed using alternate spatial placement and other parameter sensitivity tests. The model can be made more realistic by formulating parameters based on prior data, by examining sensitivity to assumptions, and by adding any other essential dimensions to the process being modelled. But the first step in exploring these relationships is formulating a simplistic toy model as Galea et al. do.

A dynamic model can ultimately be used to obtain a range of possible responses to a given intervention, allowing results to vary based on other conditions and based on uncertainties in the model. In addition, dynamic models allow the assessment of effects that may be distant in space and time. Once the model is developed, researchers can estimate short- and long-term effects of different kinds of interventions or different combinations of interventions in a virtual and completely manipulable world. This allows investigations of interventions that may not be testable in experiments and interventions implemented under conditions different from those currently observed. Most of Galea et al.'s illustration focuses on network influences under varying conditions, but networks may be complicated to intervene on. However, the inclusion of other modifiable factors such as those discussed in Cooper et al. (for example, drug-related law enforcement and presence of social/health services) would allow an examination of how intervening on these factors might modify the prevalence of addiction or its social patterning.

Even dynamic models however are not without their problems. These models also necessarily simplify reality, although they simplify in different ways than models that underlie the statistical approaches currently used in behavioural research today. As in all model development, investigators will need to determine what level of detail can be ignored for model results to be valid with respect to the main question of interest. The purpose of the model is not to replicate reality exactly but to abstract the essential elements that need to be included to answer the question at hand. The validation of these models is no easy task and there is substantial discussion on how this validation can be accomplished (Grimm \& Berger, 2006; Oreskes, 1998; Rykiel, 1996). Thus a major challenge in these models is moving beyond interesting games or "toy models" to useful representations of the problem of interest from which believable conclusions regarding the real world can be drawn (Boero \& Squazzoni, 2005).

Another important aspect is determining when these models yield insights different from the predictable effects of the assumptions that are part of the model by design. The utility of these approaches occurs when the simulation exercise allows us to see something that would not otherwise have been predicted (and which results from the dynamic relations). Otherwise these models become nothing more than self-fulfilling prophecies. Of course unpredictable results present the additional challenge of identifying whether they are artefacts (or bugs) of the model or really reflect a meaningful result that the modelling allowed us to discover.

Even if these models are difficult to make realistic enough for their results to be convincing, the simple act of specifying these models forces researchers to think about the processes likely to be involved in the problem they are studying. This is a welcome sign in a field in which the methods used (essentially focused on isolating associations) have constrained the types of research questions that are asked, and emphasis has imperceptibly shifted from understanding processes to isolating statistically independent associations. As noted by Galea et al. and others (Auchincloss \& Diez Roux, 2008; Page, 2008) the process of making these models more realistic will highlight important areas where we need more data, and may point to important outstanding questions which may be addressed with traditional analytical approaches. In addition, even toy models can be useful in that they allow researchers to explore 
dynamic processes and perhaps generate new hypotheses which can then be tested using traditional approaches.

As has been noted in relation to the incorporation of multilevel analysis into social science research (Mason, 1995) the introduction of new methods is often greeted with great enthusiasm because they appear to solve nagging problems with current approaches, this optimism is followed by disappointment as it is recognized that the methods do not deliver on all their promises, and finally this disappointment evolves into recognition that the methods do help with some things but do not solve all the problems and their impact is not as radical as initially thought. The introduction of dynamic models into health behaviour research is likely to follow a similar pattern. The tools we have used to study the determinants of behaviours have been necessarily crude. And yet it would be hard to argue that their use has not yielded useful information. Understanding the complicated processes involved in shaping behaviours (as well as the most effective strategies to changing behaviours) will likely require multiple types of evidence and analytical approaches. Agent-based models like those illustrated by Galea et al. and measurement strategies like those illustrated by Cooper et al. will be important pieces of our toolkit. The task at hand it to show that the use of these new approaches really yields new insights and ultimately makes a difference.

\section{References}

Ali, M., Emch, M., \& Donnay, J. P. (2002). Spatial filtering using a raster geographic information system: Methods for scaling health and environmental data. Health E Place, 8(2), 85-92.

Auchincloss, A. H., \& Diez Roux, A. V. (2008). A new tool for epidemiology: The usefulness of dynamic-agent models in understanding place effects on health. American Journal of Epidemiology, 168(1), 1-8.
Boero, R. \& Squazzoni, F. (2005). Does empirical embeddedness matter? Methodological issues on agent-based models for analytical social science. Journal of Artificial Societies and Social Simulation, 8(4). Accessed 01 November, 2008, http://jasss.soc.surrey.ac.uk/8/4/6.html

Cooper, H. L., Bossak, B., Tempalski, B., Des Jarlais, D. C., \& Friedman, S. R. (2009). Geographic approaches to quantifying the risk environment: Drug-related law enforcement and access to syringe exchange programmes. International Journal of Drug Policy, 20, 217-226.

Diez Roux, A. V. (1998). On genes, individuals, society, and epidemiology. American Journal of Epidemiology, 148(11), 1027-1032.

Galea, S., Hall, C., \& Kaplan, G. (2009). Social epidemiology and complex system dynamic modelling as applied to health behavior and drug use research. International Journal of Drug Policy, 20, 209-216.

Grimm, V., \& Berger, U. (2006). A standard protocol for describing individual-based and agent-based models. Ecological Modelling, 198(1-2), 115-126.

Koopman, J. (2004). Modeling infection transmission. Annual Review of Public Health, $25,303-326$.

Koopman, J. S. (1996). Emerging objectives and methods in epidemiology. American Journal Public Health, 86(5), 630-632.

Loomis, D., \& Wing, S. (1990). Is molecular epidemiology a germ theory for the end of the twentieth century? International Journal of Epidemiology, 19(1), 1-3.

Mason, W. (1995). Comment. Journal of Educational and Behavioral Statistics, 20 221-227.

Mujahid, M., Diez Roux, A. V., Morenoff, J., \& Raghunathan, T. (2007). Assessing the measurement properties of neighborhood scales: From psychometrics to ecometrics. American Journal of Epidemiology, 165(8), 858-867.

Oreskes, N. (1998). Evaluation (not validation) of quantitative models. Environmental Health Perspectives, 106(Suppl. 4), 1453-1460.

Page, S. E. (2008). Agent-based models. In N. D. Steven \& E. B. Lawrence (Eds.), The New Palgrave Dictionary of Economics. Palgrave Macmillan. Accessed 01 November 2008, http://www.dictionaryofeconomics.com/article?id=pde2008_A000218

Raudenbush, S. W., \& Sampson, R. J. (1999). Ecometrics: Towards a science of assessing ecological settings, with application to the systematic social observation of neighborhoods. Sociological Methodology, 29, 1-41.

Rykiel, E. (1996). Testing ecological models: The meaning of validation. Ecological Modeling, 90(3), 229-244.

Stallones, R. (1973). The epidemiologist as environmentalist. International Journal of Health Services, 3, 29-33. 